\title{
«Me están echando de mi casa». Repercusiones personales y sociales de la inseguridad residencial en Barcelona
}

\section{Miguel Solana-Solana Anna Ortiz Guitart}

Universitat Autònoma de Barcelona. Departament de Geografia antoniomiguel.solana@uab.cat; anna.ortiz@uab.cat

\section{Antonio López-Gay}

Universitat Autònoma de Barcelona. Departament de Geografia y Centre d'Estudis Demogràfics tlopez@ced.uab.cat

\section{Resumen}

Las condiciones para acceder a una vivienda en España han empeorado desde la crisis económica de 2008. El mercado de alquiler se ha convertido en la única opción para muchos grupos sociales. En el contexto español, con un nivel muy bajo de vivienda social y con contratos de alquiler de corta duración, el aumento del precio de la vivienda ha tenido un fuerte impacto. En este artículo exponemos los resultados de una investigación en Sant Antoni, uno de los barrios barceloneses que han experimentado un fuerte incremento de los precios de la vivienda y una intensa transformación urbana y social. Los resultados muestran la inseguridad residencial generalizada de la población residente. Las situaciones vividas han afectado a las personas y las familias, pero también implican un riesgo de descomposición del tejido asociativo del barrio.

Palabras clave: inseguridad residencial; gentrificación; desplazamiento; movilidad residencial; Barcelona; cambio urbano 
Abstract. "They're throwing me out of my house": Personal and social consequences of residential insecurity in Barcelona

Access to housing in Spain has worsened since the economic crisis of 2008, with the rental market becoming the only option for many social groups. Given that social housing is scarce in the country and rental contracts are of a short duration, the increase in housing prices has had a strong impact. In this article, we present the results of a study carried out in Sant Antoni, one of the neighborhoods in Barcelona that has experienced a significant increase in housing prices and a dramatic urban and social transformation. Our results reveal the general situation of residential insecurity of the resident population. The situations they have experienced have not only affected their personal and family lives, but also threaten to erode the associative fabric of the neighborhood.

Keywords: residential insecurity; gentrification; displacement; residential mobility; Barcelona; neighborhood change

\begin{aligned} & \multicolumn{2}{c}{ Sumario } \\ & 1. Introducción 5. «Terror. Yo vivo de alquiler»: \\ & 2. Marco conceptual y teórico experiencias de inseguridad residencial \\ & 3. Metodología 6. Conclusiones \\ & 4. «Sant Antoni despierta del Agradecimientos \\ & aburrimiento de los últimos años»: Referencias bibliográficas \\ & un barrio en transformación acelerada \end{aligned}

\section{Introducción}

Desde finales de la década de los noventa hasta la crisis de 2008, España experimentó una de las mayores burbujas especulativas inmobiliarias dentro del contexto europeo, con una escalada continua de los precios (Bosch Meda, 2019). La crisis económica, con sus repercusiones en términos de destrucción de empleo y mantenimiento o bajada de los salarios, tuvo un fuerte impacto sobre el conjunto de la población, y en especial sobre las familias hipotecadas (CSPJ, 2013; Foessa, 2012). La pérdida, o el riesgo de pérdida, de la vivienda se convirtió así en una de las principales preocupaciones sociales, especialmente en las áreas urbanas del país. En este contexto, en 2009 se constituyó la Plataforma de Afectados por la Hipoteca (PAH).

Desde los años 2013-2014, se asiste a una nueva escalada del precio de la vivienda que comienza en Barcelona y Madrid y se va extendiendo por el resto de los principales núcleos urbanos del país. A diferencia de la situación anterior, se endurecen las condiciones de acceso al crédito hipotecario, y una parte de la demanda se dirige al mercado de alquiler (Moreno, 2016; Mora-Sanguinetti y Rubio, 2014; Ortega et al., 2011). Un alto porcentaje de esta demanda está constituida por población joven (Módenes y Botelho-Azevedo, 2017; Módenes 
y López-Colás, 2014). La búsqueda de vivienda en alquiler presiona sobre una oferta relativamente reducida, y el precio del alquiler sube en la misma o en mayor proporción que el precio de compra.

En este artículo analizamos las transformaciones que se han producido en los últimos años en el barrio de Sant Antoni, que se ha constituido como uno de los casos más notorios de cambio sociodemográfico de Barcelona (López Gay, 2018). A este barrio han llegado nuevos vecinos/as, a la vez que otros se marchan, y se ha generado una creciente sensación de inseguridad residencial. Los contratos de corta duración y el incesante aumento del precio de alquiler provocan una situación de extrema vulnerabilidad. Esto no solo tiene múltiples efectos sobre las personas, que a menudo viven un importante estrés que repercute en su salud emocional y física, sino también sobre el tejido social y comunitario del barrio. Este trabajo forma parte de un proyecto más amplio que tiene como objetivo principal investigar los cambios sociodemográficos que se están produciendo en el municipio de Barcelona, con una especial atención a los vínculos con el acceso a la vivienda y con los cambios en los flujos migratorios y residenciales. Las preguntas parten de la constatación de estos procesos de cambio y conflicto social que aparecen repartidos por toda la ciudad. Estas dinámicas nos indican la existencia de procesos de desplazamiento territorial de ciertos grupos sociales y nos conducen a plantearnos quién podrá habitar en un futuro en la ciudad central. Dentro de este marco general, una parte de la investigación se ha enfocado en estudiar los impactos personales y sociales de la inseguridad residencial, y para ello se ha elegido el barrio de Sant Antoni como área de estudio para realizar un análisis más detallado de los datos cuantitativos y, en especial, desarrollar el trabajo de campo cualitativo.

El trabajo se estructura sobre la base de los siguientes contenidos. En el siguiente apartado, centramos el debate en conceptos y propuestas teóricas que giran en torno a la inseguridad residencial y a la repercusión personal que comporta, sobre todo en términos sociales y de salud, con especial referencia al caso español. Posteriormente, repasamos la evolución del mercado de la vivienda en España y los principales componentes que han tenido un papel importante en el incremento de los precios, fundamentalmente de alquiler, en las grandes ciudades. Cerramos este apartado exponiendo algunas características del territorio que conforma este caso de estudio. En el tercer apartado, exponemos la metodología que se ha seguido en la investigación. Aunque el proyecto general parte de una metodología mixta que combina la información de tipo cuantitativo, a partir de la explotación de fuentes de información sociodemográfica, y la de tipo cualitativo, con las entrevistas semiestructuradas como técnica principal, en este artículo se ha trabajado especialmente la información proporcionada por las entrevistas. Finalmente, los resultados obtenidos y el análisis de los mismos se presentan en dos apartados. En primer lugar, indicamos la cronología y las principales transformaciones del barrio en aspectos relacionados con el comercio, la restauración, la vida cotidiana; el tejido asociativo y, en especial, los cambios experimentados en el mercado de la vivienda. En segundo lugar, exploramos las experiencias de inseguridad residencial y su repercusión 
en términos de salud personal y social. Los aspectos que se trabajan sobre la repercusión social son cuatro: la destrucción de la red comunitaria y del tejido asociativo; la desarticulación de las redes sociales por la marcha de vecinos y amistades; los problemas que comporta la atención a las personas dependientes, y, por último, la pérdida de espacios de referencia e identidad.

\section{Marco conceptual y teórico}

\subsection{En torno a la inseguridad residencial y sus efectos en la salud y el bienestar}

La inseguridad residencial es una problemática que se ha retomado de forma clara en la actualidad y que aparece ligada de forma prioritaria a las condiciones de precariedad económica y creciente desigualdad social (Deidda, 2015). Campbell et al. (2013) indican que dicha inseguridad surge cuando los residentes no pueden planificar sus vidas con cierta antelación porque su estancia en la vivienda se ve amenazada por factores financieros, por una tenencia no estable o porque el tipo de alojamiento no se adecúa a las normas convencionales o culturales. Esto supone vivir en una situación de precariedad constante, y bajo la amenaza de tener que dejar la vivienda, con todo lo que comporta de alteración vital y familiar.

Si bien las relaciones entre vivienda y salud se han estudiado tradicionalmente desde la perspectiva de las condiciones físicas de la vivienda en términos de insalubridad, desde los años noventa aparecen diversas investigaciones (Bolívar Muñoz et al., 2016; Burgard et al., 2012; Cairney y Boyle, 2004; Downing, 2016; Nettleton y Burrows, 1998; Novoa et al., 2004; Tsai, 2015; VásquezVera, 2019) que apuntan a la conveniencia de estudiar otros aspectos, como las dificultades en el pago de la vivienda y cómo condiciona física y mentalmente a sus habitantes. En las sociedades actuales, trabajo y vivienda se convierten en dos fuentes crecientes de estrés emocional, y ello a pesar de que se disponga de ambos, debido a que las condiciones de incerteza y precariedad crecen (Burgard et al., 2012; Downing, 2016; Tsai 2015). Además, Nettleton y Burrows (1998) apuntan a que los procesos de individualización hacen asumir a las personas la responsabilidad de tener un trabajo y un techo, y cómo este hecho repercute sobre su salud. En este contexto, la vivienda aparece como un elemento de protección ante las inseguridades e incertezas de la vida moderna, en un mundo globalizado y con una creciente presencia de las fuerzas del mercado: «El hogar como refugio» (Dupuis y Thorns, 1998; Hiscock et al., 2001). Si se contempla el conjunto de situaciones residenciales, diversas investigaciones remarcan que los mayores niveles de angustia/preocupación se encuentran en las personas que viven de alquiler frente a las que tienen pagos pendientes de hipoteca (Hulse y Haffner, 2014), y de forma claramente superior en el caso de los propietarios de vivienda sin pagos pendientes (Cairney y Boyle, 2004).

España aparece asociada tradicionalmente a unos niveles de baja movilidad residencial y alta estabilidad residencial dentro del contexto europeo (Long, 1991) debido al fuerte predominio de la vivienda en propiedad (Módenes, 1998). Por consiguiente, se podría deducir que la sociedad española muestra 
una percepción de mayor seguridad residencial dentro del contexto europeo. Sin embargo, los datos muestran que en la actualidad no se puede sostener esta afirmación a causa de la mayor precariedad de las condiciones sociolaborales de la población española respecto a la media europea (Módenes, 2015; 2017). A partir de la European Quality of Life Survey (EQLS), Modenes (2017) estima que en 2011 1,2 millones de hogares estaban en riesgo de abandonar su vivienda a corto plazo por problemas económicos (Eurofound, 2012) y cita los siguientes factores clave de esta inseguridad residencial: una mayor inseguridad presente en el conjunto de la población, una mayor heterogeneidad interna de los grupos más vulnerables y un mayor peso de los mismos, en especial la población joven en las primeras etapas de emancipación y formación de familia. Por tanto, la relación entre (in)seguridad residencial y cambio de vivienda y movilidad residencial no es unívoca, y numerosos estudios dan cuenta de una relación compleja y en la que intervienen numerosos factores contextuales (Lu, 1999).

Dentro de estos grupos vulnerables, una proporción destacable corresponde a los hogares formados por personas jóvenes - menores de 34 años-, con todo lo que comporta de retraso en los procesos de emancipación (Módenes, 2017) y, especialmente, matrimonio y formación de familia, según el modelo tradicional familiar de España y otros países del sur de Europa, en el que se solapa la formación de un hogar con el acceso a la propiedad, y en el que a menudo intervenía la ayuda familiar (Módenes y Botelho-Azevedo, 2017).

Ante la emergencia social que ha supuesto el aumento de las situaciones de desahucio desde 2008, se abre una nueva línea de investigación que pone la atención en los efectos sobre las condiciones de salud física y mental que tiene dicha inseguridad residencial (Bolívar Múñoz et al., 2016; Vásquez-Vera et al., 2016; Vásquez-Vera et al., 2019). La vivienda repercute sobre la salud a través de cuatro dimensiones interrelacionadas en torno a la vivienda y al barrio (gráfico 1). Se ven a su vez condicionadas por la interacción del mercado y las políticas de vivienda, que configuran el sistema de vivienda, y por políticas macroeconómicas y sociales, como son las políticas redistributivas, de salud o laborales.

En el caso de la salud mental, los estudios señalan la mayor probabilidad de padecer depresión, ansiedad, trastorno adaptativo, estrés psicológico y riesgo de suicidio. En el caso de la salud física, ésta se manifiesta en la mala salud autopercibida y en la aparición o el agravamiento de trastornos crónicos como la hipertensión arterial o enfermedades cardiovasculares. Además de estos impactos, los estudios también señalan la adopción de conductas nocivas como son la mala alimentación, el mayor consumo de tabaco, alcohol y otras drogas y el sedentarismo (Vásquez-Vera et al., 2017).

Con datos procedentes de una encuesta propia a miembros de la $\mathrm{PAH}$ y la Encuesta de Salud de Cataluña, Vásquez-Vera et al. $(2016 ; 2019)$ indican niveles de salud mental precaria y de autopercepción de mala salud muy superiores entre miembros de la PAH frente al conjunto de la población. Hay que tener en cuenta la importancia que tienen las diferencias socioeconómicas entre 
Gráfico 1. Determinantes de la relación entre vivienda y salud

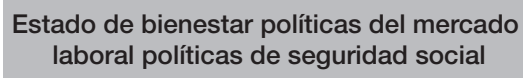

Acceso a una vivienda adecuada

\begin{tabular}{|c|c|c|c|}
\hline \multicolumn{2}{|c|}{ Vivienda } & \multicolumn{2}{|c|}{ Barrio } \\
\hline $\begin{array}{l}\text { Condiciones legales, } \\
\text { económicas y } \\
\text { emocionales (hogar) }\end{array}$ & $\begin{array}{c}\text { Condiciones físicas } \\
\text { (casa) }\end{array}$ & \multirow[t]{2}{*}{ Ambiente físico } & \multirow[t]{2}{*}{ Comunidad } \\
\hline $\begin{array}{l}\text { Asequibilidad } \\
\text { Inestabilidad } \\
\text { Vínculo afectivo }\end{array}$ & $\begin{array}{l}\text { Mala calidad } \\
\text { Hacinamiento }\end{array}$ & & \\
\hline \multicolumn{4}{|c|}{ Ejes de inequidad: clase social, género, etnia, edad y territorio } \\
\hline \multicolumn{4}{|c|}{ Impacto en salud } \\
\hline
\end{tabular}

Fuente: Vásquez-Vera (2019: 18). Marco conceptual sobre los determinantes de la relación entre vivienda y salud. Modificado de Novoa et al., 2014.

los miembros de la $\mathrm{PAH}$ y el conjunto de la población: niveles más elevados de desempleo, menor nivel educativo y una mayor proporción de trabajadores con bajos niveles profesionales. Es decir, se trata de un grupo que presenta un mayor nivel de vulnerabilidad social.

Por último, la (in)seguridad residencial en España aparece ligada a la transmisión intergeneracional de la propiedad. Las condiciones económicas pueden ser precarias en las generaciones de más edad del país debido a niveles de ingresos/pensiones bajas, pero la posesión de una vivienda en compra a la que pudieron acceder en otros momentos históricos en que se incentivó la propiedad les ofrece una situación de seguridad residencial. Sin embargo, este no es el caso de las nuevas generaciones que intentan acceder a la vivienda (Módenes, 2017; Poggio, 2008).

En este artículo incidiremos especialmente en los relatos de estos jóvenes adultos que se encuentran en un mercado de la vivienda de difícil acceso y con unas condiciones contextuales marcadas por la precariedad laboral y por las restricciones a la estabilidad del sistema legal que regula el mercado de alquiler. Son gente que vive y quiere vivir en un barrio atractivo por los servicios y equipamientos que ofrece, su localización y buen sistema de comunicaciones, y con una intensa vida asociativa y comunitaria que lo sitúa dentro del imaginario colectivo de la ciudad como una de las áreas más vivas y ricas. El crecimiento de la oferta de vivienda turística, la entrada de nuevos residentes con perfiles socioprofesionales medios y altos y una creciente presencia de inversores que buscan y compran viviendas y edificios enteros en el barrio se contemplan como una amenaza. 


\subsection{Mercado de la vivienda y factores contextuales en Barcelona}

Según datos de la European Quality of Life Survey de 2012, el 18 \% de los hogares que tenían un alquiler privado indicaron el riesgo de tener que abandonarlo de forma forzosa frente al $9 \%$ de los hogares con vivienda pendiente de pago de hipoteca (Módenes, 2017). Estos datos se confirman en estudios posteriores en que se controlan las características sociodemográficas de los hogares (López-Colás et al., 2018). Otro hecho significativo que afecta a la vivienda en alquiler es la reforma de la Ley de Arrendamientos Urbanos (LAU), promulgada en 1994 y reformada en 2013, que, entre otras modificaciones, redujo el período mínimo de los contratos de cinco a tres años. La LAU, así como otras leyes que estructuran y conforman el contexto regulatorio del mercado de la vivienda en España, es asimismo un factor de inseguridad residencial (Módenes y López-Colás, 2014).

Barcelona cuenta con una oferta de vivienda en alquiler significativamente superior a la media española, y más elevada que otras grandes urbes del país (un $38 \%$ de la población vive en régimen de alquiler en el municipio, según la Encuesta Sociodemográfica de Barcelona de 2017). Ahora bien, el porcentaje de vivienda social de alquiler representa solo un 1,4\% (PAH y ASPB, 2018) del parque residencial, y responde al modelo dual que propone Kemeny (1995), en el que hay un bajo porcentaje de alquiler social que no se traduce en la posibilidad de influir en el mercado de alquiler privado, lo que lleva a que la mayoría de la población opte por la propiedad de la vivienda como mecanismo de protección. Asimismo, tres factores presionan en el fuerte aumento de los precios de alquiler en los últimos años.

Por una parte, el incremento de la llegada de turistas a la ciudad (Cócola Gant, 2016; Mansilla y Milano, 2019), y con ello la transformación de una parte significativa de la oferta de vivienda de alquiler para población residente a oferta de alojamiento para turistas. Diversos estudios muestran la diferencia de rentabilidad que se puede obtener si la vivienda se dirige al turismo (Garcia-López et al., 2019). Barcelona se ha convertido en una de las principales proveedoras de alojamiento turístico a través de la plataforma Airbnb. Y ello se ha producido a pesar de que en los últimos cuatro años se ha llevado a cabo desde el ayuntamiento una política de freno a este tipo de empresas y de persecución de la oferta de vivienda para alquiler turístico no regularizada (Blanco-Romero et al., 2018). En esta creciente presión sobre el mercado de la vivienda de alquiler, otro factor es la atracción que tiene la ciudad para la llegada e instalación de población con niveles altos de estudios y cualificación profesional, que en muchos casos es de origen extranjero y que presenta un fuerte componente de transitoriedad (López-Gay, 2016; 2017). En los últimos años se constata la instalación en la ciudad de un número creciente de empresas de nuevas tecnologías y sectores económicos emergentes, y la atracción de mano de obra extranjera cualificada (Sorando y Leal, 2019).

Por último, a estas transformaciones se ha sumado la entrada de particulares, pero especialmente de fondos de inversión y otro tipo de instrumentos 
Gráfico 2. Localización del barrio dentro de la trama urbana del municipio de Barcelona

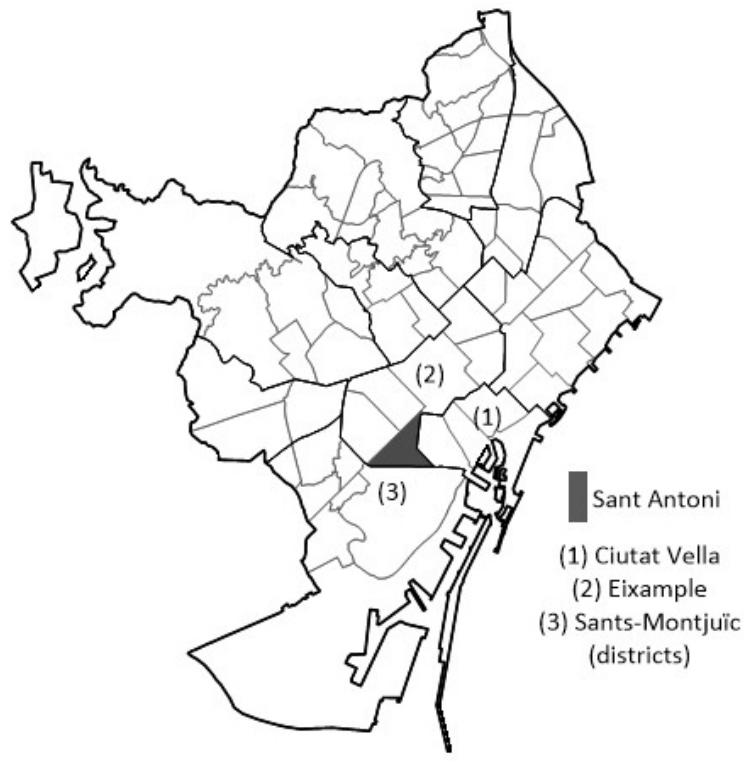

Fuente: elaboración propia.

financieros, en el mercado de alquiler. La caída del precio de la vivienda tras la crisis de 2008, junto con la imposibilidad de una parte de la población de comprar debido al endurecimiento de las condiciones de crédito, generó un escenario propicio que convirtió la vivienda de alquiler en un sector con elevados niveles de rentabilidad, un fenómeno que se ha observado en otras muchas ciudades globales (Fields y Uffer, 2014; Wijburg et al., 2018).

Según datos del ayuntamiento de Barcelona, el precio medio del $\mathrm{m}^{2}$ en alquiler para el conjunto de la ciudad se ha encarecido un $39 \%$ entre 2013 y 2019 , de $682 €$ a $944 € .{ }^{1}$ Sant Antoni, el barrio que analizamos en este trabajo, se sitúa ligeramente por encima (986 €), con un encarecimiento en línea con el experimentado por el conjunto de la ciudad. Desde la crisis económica, y especialmente como deriva del movimiento $15 \mathrm{M}$ del año 2011 , surgen numerosas plataformas y asociaciones. Dentro de esta eclosión de grupos que luchan contra la gentrificación y la exclusión residencial, en 2017 se constituyó el Sindicat de Llogateres (Sindicato de Inquilinas), que, tomando como modelo la $\mathrm{PAH}$, se erige en plataforma de defensa de los inquilinos/as.

1. Portal estadístico del Ajuntament de Barcelona (<bcn.cat/estadística $>$ ). Consulta realizada el 25/09/2019. La fuente de datos proviene de la Secretaria d'Habitatge i Millora Urbana (Generalitat de Catalunya) a partir de las fianzas de alquiler depositadas en el Incasòl. 
Sant Antoni es uno de los 73 barrios de Barcelona. Está situado en el distrito del Eixample de Barcelona y tiene una población de 38.182 habitantes (Barcelona: 1,6 millones), una extensión de 80,5 hectáreas (Barcelona: $10.135,3 \mathrm{ha}$ ) y una densidad de $479 \mathrm{hab} . /$ ha (Barcelona: 160). Si bien no forma parte del conjunto de barrios que conforman el casco histórico de la ciudad (Ciutat Vella), es colindante a esta área y se encuentra a poca distancia del frente marítimo y de una de las principales zonas verdes de la metrópoli: Montjuïc (gráfico 2). Aunque no dispone de un patrimonio arquitectónico o cultural especialmente atractivo para el turismo, las edificaciones se construyeron mayoritariamente a finales del siglo XIX, y la estructura urbana de calles y aceras amplias y rectas fruto de la planificación heredera del Pla Cerdà permite disponer de un parque residencial caracterizado por viviendas de superficie media/grande y relativamente bien equipadas.

\section{Metodología}

Para el planteamiento del conjunto de la investigación, se ha seguido una metodología mixta de tipo convergente. Se combina la explotación cuantitativa (hemos utilizado los censos de población de 2001 y 2011, la Encuesta Sociodemográfica de Barcelona de 2017 y datos sobre la evolución del precio de la vivienda) con un trabajo de campo de tipo cualitativo basado en la observación y la participación. Esta labor ha consistido en la participación en asambleas y encuentros con algunas entidades del barrio, pero sobre todo en la realización de entrevistas semiestructuradas a los colectivos que a continuación se presentan. Las entrevistas nos permiten profundizar en el interés inicial que teníamos por conocer las experiencias de las personas que viven o han vivido en el barrio y en cómo perciben su transformación. Además, nos ofrecen la posibilidad de recoger de forma fidedigna y cargada de significados y matices las vivencias y los sentimientos que genera el hecho de sentirse expulsado de la propia vivienda/barrio, con todo lo que comporta de carga emocional y afectación personal. El trabajo de campo se llevó a cabo durante el segundo semestre de 2018. Se entrevistó a un total de 33 personas (19 mujeres y 14 hombres) pertenecientes a tres grupos distintos (tabla I).

La edad de los vecinos/as que llevan menos tiempo residiendo en el barrio es menor, además son personas con niveles de formación más elevados, con una mayor proporción de extranjeros. Generalmente, comparten vivienda con otras personas. Mientras que los adultos más mayores — todos españoles- pertene-

Tabla I. Personas entrevistadas.

\begin{tabular}{lcrr}
\hline & Hombres & Mujeres & Total \\
\hline Residentes con más de 10 años viviendo en el barrio (RES) & 4 & 4 & 8 \\
Residentes llegados en los últimos 10 años (IN) & 8 & 7 & 15 \\
Personas que se han marchado del barrio (MAR) & 2 & 8 & 10 \\
Total & 14 & 19 & 33 \\
\hline
\end{tabular}

Fuente: elaboración propia. 
Tabla II. Perfil de las personas entrevistadas

\begin{tabular}{|c|c|c|c|c|}
\hline & RES & IN & MAR & Total \\
\hline \multicolumn{5}{|l|}{ Sexo } \\
\hline Mujeres & 4 & 7 & 8 & 19 \\
\hline Hombres & 4 & 8 & 2 & 14 \\
\hline \multicolumn{5}{|l|}{ Edad } \\
\hline Menores de 30 años & 0 & 4 & 4 & 8 \\
\hline $31-40$ & 2 & 8 & 4 & 14 \\
\hline Mayores de 40 años & 6 & 3 & 2 & 11 \\
\hline \multicolumn{5}{|l|}{ Nivel de estudios } \\
\hline Secundaria & 5 & 0 & 1 & 6 \\
\hline Estudios superiores (grado) & 3 & 6 & 4 & 13 \\
\hline Estudios superiores (posgrado) & 0 & 9 & 5 & 14 \\
\hline \multicolumn{5}{|l|}{ Nacionalidad } \\
\hline Española & 8 & 9 & 9 & 26 \\
\hline Extranjera & 0 & 6 & 1 & 7 \\
\hline \multicolumn{5}{|l|}{ Composición del hogar } \\
\hline 1 persona & 1 & 1 & 3 & 5 \\
\hline 2 personas & 2 & 3 & 2 & 7 \\
\hline 3-4 personas & 5 & 3 & 2 & 10 \\
\hline Comparte piso & 0 & 8 & 3 & 11 \\
\hline Tenencia & 4 & 5 & 2 & 11 \\
\hline \multicolumn{5}{|l|}{ Piso de propiedad } \\
\hline Piso de alquiler & 4 & 10 & 8 & 22 \\
\hline
\end{tabular}

Fuente: elaboración propia a partir de las entrevistas realizadas.

cen al grupo que lleva más tiempo residiendo en el barrio, no comparten piso y no tienen estudios tan altos como los recién llegados (tabla II).

El guión de entrevista giró alrededor de la historia residencial, el sentido de pertenencia, la vida cotidiana, la red de relaciones y la vinculación con el tejido asociativo del barrio, la valoración del barrio, la visión de la transformación del barrio y las perspectivas de futuro. Las entrevistas se realizaron mayoritariamente en distintas ubicaciones del barrio (domicilios particulares y espacios públicos o cafeterías), y duraron alrededor de una hora. Se grabaron, se transcribieron en su totalidad y fueron codificadas con Atlas-ti.

Para complementar las opiniones y valoraciones de los vecinos/as entrevistados, se realizaron entrevistas a miembros de la Associació de Veïns i Veïnes de Sant Antoni y del colectivo Fem Sant Antoni. Durante el trascurso del trabajo de campo, asistimos de forma regular a las asambleas, abiertas al público, de este colectivo y del Sindicat de Llogateres, que lucha contra los procesos de expulsión de vecinos del barrio y contra los procesos especulativos que se están produciendo.

En este artículo hemos utilizado el conjunto del material recogido en el trabajo de campo, ya que las referencias al tema de la vivienda constituyen el eje 
principal de todas las entrevistas. Además, todas las personas entrevistadas, con independencia de su edad, origen, situación residencial (propiedad o alquiler) o lugar de residencia en el momento de la entrevista (recién llegados, personas que se han marchado y residentes de toda la vida), hablan ampliamente sobre la situación del barrio, la transformación social que observan y los problemas de acceso a la vivienda, ya sea de compra o alquiler, y la propia inseguridad residencial. Sin embargo, nos concentramos en aquellos entrevistados que vivían o todavía viven de alquiler. En el caso de los que se vieron forzados a abandonar el barrio, fueron relatos muy vivos y en algunas ocasiones dolorosos, lo que demuestra la profundidad de las heridas del proceso de "expulsión", tal como ellos y ellas lo han expresado. En el caso de los residentes en el barrio que viven de alquiler, incluyendo los que han llegado durante los últimos 10 años y que también viven de alquiler, se detecta la preocupación e incerteza que provoca vivir en el barrio, con todo lo que comporta de amenaza para sus proyectos vitales. $^{2}$

\section{4. «Sant Antoni despierta del aburrimiento de los últimos años»: un barrio en transformación acelerada}

Este es el titular que la revista Time Out publicó en abril de 2015, que contenía como reportaje central el cambio que estaba experimentando el barrio. Destaca la novedad de locales que "aportan un aire fresco al barrio" y remarca que estos conviven con algunos de toda la vida. Sin duda, el epicentro es la calle Parlament, que con tan solo tres manzanas se ha convertido en «la calle de los 37 bares». ${ }^{3}$ Las aperturas en 2011 del Bar Calders y en 2013 del Bar Federal son momentos trascendentales en la transformación del paisaje urbano del barrio.

Otra fecha significativa en esta breve historia del proceso de transformación del barrio y sus habitantes son los años 2017-2018, cuando se produce el vaciado del edificio situado en la calle Parlament 1-3. Es un momento importante dentro del proceso de toma de conciencia del proceso de expulsión de vecinos y vecinas del barrio. Fem Sant Antoni, una asociación de defensa del barrio, nació en 2016 y lo hizo con la voluntad de luchar contra el impacto que tiene el turismo sobre el comercio, la vivienda y el espacio público. ${ }^{4}$ Sin embargo, en 2017, dentro de la asociación se constituye un grupo dedicado exclusivamente a los problemas derivados de la vivienda debido a la entrada constante de consultas sobre este tema. Desde esa fecha, prácticamente toda la actividad del colectivo se ha centrado en el tema de la vivienda y los procesos de expulsión de población, ya sea a través de desahucios o de aumentos y no renovación de los contratos de alquiler.

2. Citas: nombre ficticio, edad y un código según las categorías establecidas en esta investigación (RES, IN y MAR).

3. El Pais, 11/11/2017.

4. <https://femsantantoni.wordpress.com/>. 
Para las personas entrevistadas, el tejido comercial del barrio no para de cambiar: nuevos restaurantes, bares, peluquerías, panaderías, comercios de pasta fresca, tiendas de ropa infantil, de cómics, entre otros ejemplos, con una gran profusión de comercios de franquicia y la sensación de que los comercios de proximidad se cierran en un claro ejemplo de gentrificación comercial que también ha afectado a otros barrios céntricos de la ciudad (Hernández Cordero, 2016; Hernández Cordero et al., 2016). Así lo manifiesta Marc:

Se ha perdido el comercio, la vida que había alrededor del mercado, así lo comenta la gente mayor. Y cuando me encuentro con amigos de mis padres y les dices «¿qué tal?», en general dicen: «Fatal, a ver cuando nos lo vendemos y nos vamos». Es que es otro barrio, no es lo mismo. (RES_Marc, 37 años)

Finalmente, en mayo de 2018 se inaugura la reforma del mercado central del barrio. Para las personas entrevistadas, la apertura del nuevo mercado se percibía como un elemento atractivo y a la vez una amenaza. Temen que el espacio llegue a parecerse demasiado al principal mercado de la ciudad, La Boqueria. Incluso se utiliza el término «boquerización» por parte de algunas personas entrevistadas para describir el proceso de transformación de un mercado cuando este pierde la función de dar servicio y provisión de alimentos a la población que vive en el barrio y se transforma en un lugar para el consumo turístico.

Pero sin lugar a dudas, el cambio más importante, general en toda Barcelona, se produce en el mercado de la vivienda. Por un lado, el alquiler gana terreno al régimen de tenencia en propiedad, sobre todo en edades adultas jóvenes. En 2017, casi el $60 \%$ de la población de 25 a 49 años residente en Sant Antoni era inquilina, una cifra cinco puntos porcentuales por encima de la media de Barcelona y muy superior a la registrada en los censos de 2001 y 2011 (tabla III). Por otro lado, desde 2013 se experimenta un fuerte aumento en el precio de la vivienda, tanto de compra como de alquiler. En el gráfico 3, se presenta el valor medio de la ciudad, de Sant Antoni y del resto de barrios de Barcelona agrupados en cuartiles según la renta media por persona (según los datos de renta del Instituto Nacional de Estadística de 2016). Los precios de Sant Antoni se sitúan ligeramente por encima de la media del municipio en el caso del mercado de compra y en valores similares en el de alquiler. Los precios de compra en Sant

Tabla III. Evolución de la proporción de personas que viven de alquiler. Sant Antoni y Barcelona, 2001-2017.

\begin{tabular}{ccccccc}
\hline & \multicolumn{2}{c}{ Sant Antoni } & & \multicolumn{2}{c}{ Municipio de Barcelona } \\
\cline { 2 - 3 } \cline { 5 - 6 } & $25-49$ años & Toda la población & & 25-49 años & Toda la población \\
\hline 2001 & $37,0 \%$ & $33,3 \%$ & & $26,3 \%$ & $28,5 \%$ \\
2011 & $47,2 \%$ & $36,0 \%$ & & $39,3 \%$ & $29,9 \%$ \\
2017 & $58,3 \%$ & $43,9 \%$ & & $53,7 \%$ & $37,6 \%$ \\
\hline
\end{tabular}

Fuente: elaboración propia a partir de censos de población de 2001 y 2011 y Encuesta Sociodemográfica de Barcelona de 2017. 
Gráfico 3. Evolución del precio de la vivienda de alquiler (izquierda) y compraventa de segunda mano (derecha) en Sant Antoni y en el resto de barrios de Barcelona (según cuartiles de renta, 2016), 2006-2018.

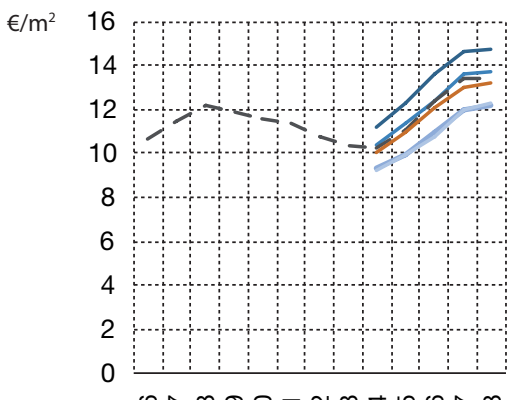

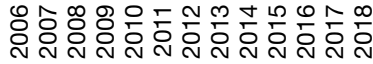

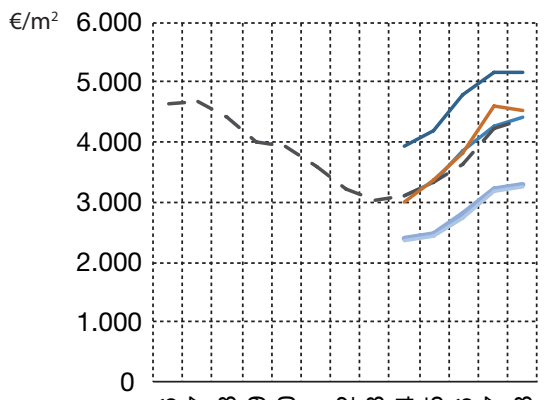

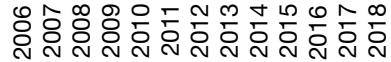
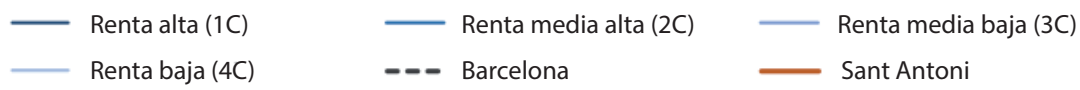

Fuente: elaboración propia a partir de: (i) alquiler: Secretaria d'Habitatge i Millora Urbana, a partir de les fianzas de alquilar depositadas en el Incasòl; (ii) compra: Idealista.com (informe anual). Web del Departament d'Estadística, Ajuntament de Barcelona.

Antoni han crecido un $51 \%$ entre 2014 y 2018, por un $40 \%$ en el conjunto de la ciudad, y los del alquiler un $32 \%$, por un $31 \%$ en la ciudad. Francisco compró un piso en Sant Antoni en el año 2013, en un momento de inflexión de los precios de la vivienda en la ciudad hacia una tendencia alcista. Es consciente de que fue una buena compra en ese momento, y asiste desde entonces a una rápida escalada de los precios que ha hecho, por ejemplo, que esté recibiendo ofertas de compra constantemente. No obstante, su intención es quedarse en el piso y en el barrio, donde ya se encuentra asentado y con una red social y de conocimiento de comercios y espacios sociales consolidada. Sin embargo, se han ido marchando los vecinos que vivían en pisos de propiedad, ya que les ofrecían cantidades importantes de dinero. Ninguno se ha quedado en el barrio.

Fue una buena compra porque era muy barato. La misma inmobiliaria que nos lo vendió cada dos o tres meses nos llama para decirnos que tiene clientes, y nos ha llegado a ofrecer tres veces lo que saben que pagamos, independientemente de que hemos hecho reformas. Es una locura total (...). Y alquilados, antes de acabar la reforma, pisos que no llegan a 50 metros por más de $1.000 €$ (...). Ya en la última llamada, de hará dos o tres meses, me dijo: «Es que tengo un cliente que tiene tanto». Le digo: «Es que no me interesa». Me dice: "Cuánto te interesa». "Es que no me interesa irme del barrio». «Pero tú di un número, porque todo tiene un número». (IN_Francisco, 39 años)

La presión ha llegado a tal extremo que coge fuerza el volumen de alquileres que no tienen como objeto la vivienda sino el alquiler de habitaciones. En 
una información facilitada por Fem Sant Antoni en una reunión del colectivo celebrada el 3 de marzo de 2018, se comunicó la existencia de un bloque de apartamentos-vivienda en la calle Floridablanca 80 que se alquilaba por habitaciones a un precio de $700 €$ / vivienda, lo que representaba $2.800 €$ / apartamento / mes.

Hay muchísima demanda de habitaciones porque la gente ahora mismo no puede pagarse un piso entero, y también se puede sacar mucho provecho de alquilar una habitación... Igual si lo hicieras proporcionalmente deberías pagar 300 y te están cobrando 600 por una habitación. La gente necesita encontrar un lugar donde vivir. (MAR_Noemí, 36 años)

Estas transformaciones no son nuevas. Ya en la década de los años noventa se vivió una situación similar en la que toda una generación de personas jóvenes - mayoritariamente nacidas en la época del baby boom en España- no pudieron acceder a la vivienda en el barrio debido a la escasez de oferta y los altos precios. En ese momento esto ya tuvo un impacto importante sobre el tejido asociativo del barrio, tal como comenta Mateu, que vivió como una gran pérdida la descomposición del grupo de amigos que se había forjado y que participaban en proyectos comunes. El pudo quedarse en el barrio en aquel momento porque recibió un piso en herencia.

Puedo hablar de una veintena de personas que habíamos crecido juntos en el barrio, muy metidos en la vida asociativa vecinal, y solo dos parejas de todo este grupo nos quedamos a vivir en el barrio. El resto se marchó. Y eso estoy hablando de finales de los años noventa y mediados del año 2000 (...). Fuimos muy afortunados, y si no hubiéramos tenido esta herencia familiar, difícilmente nos hubiéramos quedado a vivir en el barrio (...). Compañeros que habían creído en el proyecto de barrio, que habían tejido muchas complicidades..., poco a poco toda esta gente fue desapareciendo. Lo vivimos bastante dramáticamente. (MAR_Mateu, 45 años)

Esta situación causa una serie de repercusiones personales y sociales en la gente que habita en el barrio, tal como expondremos a continuación. Una de las más importantes es la sensación de incertidumbre e inseguridad que padecen especialmente las personas que están de alquiler, ya que perciben claramente su situación como más vulnerable debido a la combinación del alza de precios con la existencia de contratos de corta duración.

\section{5. «Terror. Yo vivo de alquiler»: experiencias de inseguridad residencial}

Los procesos de inseguridad residencial tienen repercusión especialmente en los más vulnerables: personas mayores, mujeres solas y con dependencias familiares. La precariedad laboral y económica se suma a las cargas familiares. Esta situación de vulnerabilidad la sienten tanto las personas que se han ido del barrio como los nuevos vecinos/as que se han instalado en él - especialmente si están con un contrato de alquiler y si viven en un edificio 
de propiedad vertical. Las palabras que se han utilizado para referirse a los sentimientos que provoca la situación de inseguridad residencial son: vulnerabilidad, desprotección, estrés, año infernal, pesadilla continua, desestabilizador, miedo, angustia, rabia, impotencia, sufrimiento e incertidumbre. Lina vivió una situación muy estresante cuando le dijeron que no le renovaban el contrato del piso y que en unos meses tenía que abandonarlo.

Me da mucha pena porque ves que ha habido un cambio de gente, y yo creo que en el $90 \%$ de los casos no ha sido voluntario. Ves otro nivel de vida, otro estatus de la gente que vive porque son los que pueden permitirse pagar $1.500 €$ por un piso de mierda. (MAR_Lina, 31 años)

Los efectos sobre las personas tienen un marcado componente emocional, aunque en algún caso se han mencionado también problemas físicos como consecuencia de la situación de tensión y sufrimiento que se vive durante el proceso de acoso inmobiliario (Hiscock et al., 2001; Hulse y Saugeres, 2008; Nettleton y Burrows, 1998). Un amplio estudio realizado por la PAH y ASPB (2018) en Barcelona indica que el aislamiento y la sensación de pérdida de elementos cotidianos de referencia, como son los cambios en el comercio o en la red de amistades y asociativa, así como la falta de apoyo social para afrontar imprevistos, impactan en la salud física y mental de las personas que se encuentran en situación de inseguridad residencial. Una mujer activista en la asociación Fem Sant Antoni que se mudó al barrio recientemente y vive de alquiler apunta que no puede abandonar la situación de incertidumbre acentuada por la poca duración de los contratos de alquiler.

Terror. Yo vivo de alquiler, tengo contacto con la propietaria, tengo una buena relación. Creo que está contenta con nosotros como inquilinos. Ambos trabajamos, no damos problemas. Y aun así tengo miedo, aunque tengo una situación en mi caso privilegiada. Vives constantemente con el miedo. (Mujer activista de Fem Sant Antoni)

Otro hombre activista de Fem Sant Antoni comentó en la misma entrevista: «Una persona me dijo: "No puedo deshacer las maletas"». Roger decidió venir a vivir al barrio y encontró piso en un edificio de propiedad vertical que comparte con amigos. No quiere irse ahora mismo del piso ni del barrio, pero la situación en su caso también es de inseguridad. El problema es que la dinámica de precios en el mercado de alquiler hace que cada vez se complique más la búsqueda de una vivienda a la que pueda acceder, especialmente si busca en un determinado barrio donde se plantea un proyecto de vida y se tiene ya una red social y comunitaria.

Tengo ganas de quedarme hasta que quiera, pero no depende de nosotros (...). La sensación es que pueden hacer lo que quieran. Me costaría marchar porque me he adaptado bien al barrio, me gusta. El piso me gusta. Creo que, con los precios que hay actualmente, el piso está muy bien porque, insisto, no fue fácil 
encontrarlo. Es una guerra. Cuando yo busqué era complicado encontrar un piso en Barcelona. Ahora es que ni quiero imaginarlo. (IN_Roger, 30 años)

Además, diversas investigaciones muestran que la estabilidad residencial se asocia a un impacto positivo no solo para el propio individuo, sino también para su entorno familiar y comunitario: posibilidad de desarrollar planes vitales, valor cívico más elevado, comunidades urbanas locales más estables e integradas y mejores resultados educativos infantiles (Campbell et al., 2013; Chan y Tweedie, 2015; Módenes y López-Colas, 2014).

La situación de angustia y estrés que comentan algunas de las personas entrevistadas llega a ser de tal magnitud que la salida del piso y del barrio se contempla como una liberación: "Consiguieron que hasta quisiera huir» (MAR_Sonia, 29 años). Así lo comenta Lina después de vivir lo que ella denomina un "auténtico infierno». Y no es solamente que se quiera dejar el piso, sino que va más allá: el sentido de pertenencia ya no es el mismo, se ha ido rompiendo a medida que ha ido creciendo el sentimiento de desprotección e inseguridad. A pesar de ello, todavía hoy dice estar muy afectada porque considera que tuvo que irse de un lugar que consideraba propio:

Me decía que me estaban haciendo un favor haciéndome marchar de aquí porque el barrio se ha convertido en algo que no sé qué es (...), me da pena lo que ha pasado con el barrio, porque creo que ha dejado de ser el barrio que era. Es extraño cuando paso por el barrio porque no lo siento, pero lo sigo sintiendo como mi barrio. Es como una sensación un poco de desapego. Por un lado, pienso que son mis calles. Te cruzas con guiris y me digo: «Es mi barrio no es el tuyo». Tengo una rabia interna. Pero por otro lado, lo veo tan diferente. Lo echo de menos pero ya no es lo que era. Y da mucha rabia, mucha pena. (MAR_Lina, 31 años)

En las entrevistas realizadas las referencias a las repercusiones sociales y comunitarias que provoca la sensación de inseguridad residencial son numerosas. Nosotros las hemos clasificado en cuatro tipos. En primer lugar, aparece la idea de la paulatina destrucción de la red comunitaria y de tejido asociativo que se ha ido conformando en el barrio a lo largo de los años, y que le da un carácter propio que permitía arraigar con más facilidad y crear sentimientos de pertinencia. El barrio cuenta hasta el momento con un tejido asociativo rico y constituye una referencia dentro de la ciudad.

El barrio, yo como lo viví en mi juventud, tal vez mantenía cosas que en otros barrios de Barcelona se habían ido perdiendo. Un vínculo vecinal muy curioso. Yo creo que aún vive de esta inercia, muy popular y con un tejido asociativo muy rico y muy cohesionado en el sentido de pertenencia a un barrio. (MAR_Mateu, 45 años)

La pérdida de tejido asociativo y vecinal se contempla como un tipo de vulnerabilidad que, junto con la situación de incertidumbre en que vive la población, provoca malestar y desconcierto. 
Se están haciendo cosas muy chulas y sufro y tengo miedo cuando gente que tiene ganas no se puede quedar a vivir aquí. Por ejemplo, los ocho compañeros que te he dicho están compartiendo piso entre ellos. El día que mañana se quieran ir a vivir solos con las parejas, no sé si podrán encontrar piso. Ocho personas implicadas en el barrio que hacen cosas en el barrio tal vez se tendrán que marchar. (MAR_Pep, 31 años)

Es un tema comentado por los vecinos/as que se han ido del barrio, pero también queda recogido en las preocupaciones de los nuevos vecinos/as que se han instalado en él y de los residentes de toda la vida.

Lo que me gusta más es que la zona conserva aún lo que aquí llaman la vida de barrio, que realmente los vecinos se reúnen y organizan tanto las fiestas como algún que otro evento cultural, y eso a mí me parece muy agradable. (IN_Pascal, 31 años)

En segundo lugar, se contempla la posible marcha de las amistades y la desarticulación de las redes sociales que se han creado en el barrio y que constituyen un importante elemento de apoyo. Marc, un nuevo vecino que compró un piso en el barrio en 2014, comenta:

Mis mayores preocupaciones son que la mayoría de mis amigos están de alquiler. Mi miedo es encontrarme en tres años sin un amigo al lado. Porque lo que está pasando con los alquileres es impresionante (...). Es una lástima porque ya conoces a la gente de los bares, de los súpers, los vecinos, y cuando has hecho una minired y te sientes cómodo debes marcharte. (IN_Marc, 36 años)

Son constantes las referencias a la importancia del barrio y la vida de barrio, que podríamos situar como una zona intermedia entre el espacio privado que constituye el hogar y la ciudad. Igual que se asocia un componente de protección y seguridad a la vivienda, dichas características también se trasladan al barrio. Un barrio que se reconoce en sus elementos estructurales, como puede ser el comercio, pero también en las personas que circulan por sus calles y que confieren a sus vecinos/as una sensación de seguridad. En este sentido, varios entrevistados hacen referencia al concepto de «vida de pueblo» para caracterizar el sentimiento de proximidad y comunidad que creen que todavía mantiene Sant Antoni.

Hay un sentimiento de barrio bastante fuerte. Yo he visto gente que hace muchos años que vive aquí que no quiere irse. Esto en otros barrios de Barcelona quizás no se ve tanto, y aquí lo he visto. (IN_Roger, 30 años)

En Cataluña, como en otros países del sur de Europa, el cuidado de las personas y la protección social pasa básicamente por la familia y el apoyo mutuo que proporciona, por lo que es importante la proximidad a familiares y amistades (Esping Andersen, 1990; Flaquer, 2004; Módenes, 2017). En 
este sentido, la pérdida de las redes sociales es un obstáculo para atender a las personas dependientes. La dificultad que tienen las generaciones jóvenes para encontrar vivienda comporta que tengan que marcharse y alejarse de sus padres, que envejecen y cada vez son más dependientes de los hijos.

Por otra parte, en los discursos recogidos aparece el tema de los hijos, que ya están escolarizados en el barrio en colegios cercanos y que tienen sus redes de amistad y sus espacios de referencia en el barrio y en su entorno más próximo. Este es un elemento que enraíza a los vecinos al barrio y que hace más problemático el proceso de salida. Desde Fem Sant Antoni se apunta a la angustia que se ha traspasado de los adultos hacia los menores (Hulse y Saugeres, 2008), quienes empiezan ya a reproducir los discursos de incertidumbre sobre la vivienda:

Casi todo el mundo que ha pasado por aquí tienes hijos. Con el cambio, el hijo pierde la escuela, los amigos, el esparcimiento. Es una de las cosas que la gente vive con más angustia (...). La gente de las ampas contactó con nosotros porque es un tema que se habla en los patios. Es un problema porque los niños se están marchando. (Hombre activista de Fem Sant Antoni)

Un cuarto elemento que se ha mencionado en las entrevistas es la pérdida de los espacios de referencia e identidad, que en muchos casos van ligados a las prácticas de ocio. Lugares que formaban parte de la cotidianidad se transforman por la afluencia de un nuevo tipo de público. Y esto se suma a la transformación del paisaje comercial, que muchos entrevistados/as comentan.

Para mí, es paradigmática la Bodega d'en Rafel. Una bodega que era, para hablar así claro, un antro fantástico donde iban los borrachos del barrio. En estos momentos puedes ver cómo se está convirtiendo en un bar hipster. (Hombre activista de Fem Sant Antoni)

El incremento del precio de la vivienda, la marcha por la subida de los precios de alquiler y la trasformación social y comercial que experimenta el barrio también se ponen con relación a los que quedan, normalmente propietarios de la vivienda. El barrio se transforma en un lugar distinto, que puede resultar extraño y hostil para los vecinos/as que lo habitan, donde pueden sentir que la red social y comunitaria que les ha servido de apoyo y referencia a lo largo de la vida se ve afectada o destruida por estos cambios. Desde Fem Sant Antoni apuntan que la sustitución de población parece inevitable en este contexto:

La gente propietaria también sufre porque ve que va perdiendo los vecinos. Una persona mayor acaba marchándose. Acaba vendiendo el piso porque no tienen la red que ha tenido siempre. Además, las ofertas son muy bestias (...). ¡A toda esta gente le están cambiando el barrio! (Hombre activista de Fem Sant Antoni)

Una de las respuestas posibles ante la inseguridad residencial es la opción de comprar una vivienda, lo que permite amortiguar la sensación de vulne- 
rabilidad. $\mathrm{Y}$ en algunos casos, la solución ha pasado o está previsto que pase por la trasmisión intergeneracional de la vivienda (Módenes, 2017; Poggio, 2008). De todas formas, es una opción limitada a aquellos hogares con la suficiente capacidad económica, por ahorros o ingresos, para poder entrar en un mercado hipotecario crecientemente selectivo. Clara vivía en un piso de alquiler en otro barrio de la ciudad, pero decidió comprarse un piso con su pareja gracias a unos ahorros y a la ayuda de los padres. Con esta decisión, dice: «Nos libramos de este padecimiento, de estar pendientes de si nos suben o no» (IN_Clara, 42 años).

Marta también consideró la opción de comprar ante la escalada de precios de los pisos de alquiler. Compró porque consideró que era la mejor alternativa a continuar alquilando. El piso le costó $96.000 €$ y ahora cree que podría venderlo por $150.000 €$. Piensa que fue una decisión acertada, y más viendo el panorama actual:

Comprar este piso fue un golpe de suerte porque era prácticamente imposible comprar, no solo por los precios, sino porque lo que yo buscaba es lo que se llama pisos de inversión, que eran los de menos de $100.000 €$. Ahora eso ya no existe. Yo iba a la inmobiliaria, y te decían: «¿Lo quieres para inversión?» $Y$ yo: «No, lo quiero para vivir». Les extrañaba que estuvieras buscando un piso pequeño o de ese precio para vivir (...). Y competías con inversionistas, con gente extranjera y con gente que además no van a fijarse mucho en ciertas cosas del piso porque no van a vivir ahí. (IN_Marta_30 años)

\section{Conclusiones}

El acceso a la vivienda presenta cada vez más dificultades, especialmente para la población joven que se emancipa y para los grupos sociales con menos recursos económicos. Trabajos a menudo precarios, con poca estabilidad y con sueldos bajos, se unen a un mercado de la vivienda que se encarece, especialmente en los principales centros metropolitanos. Desde 2013 se asiste a una recuperación del precio de la vivienda, tanto de compra como de alquiler, pero el endurecimiento de las condiciones del crédito ha hecho que el alquiler sea la única opción para determinados grupos de población.

En el caso que hemos analizado, hemos querido incluir no solo a los residentes que de forma permanente o de forma prolongada han vivido en el barrio, sino también a aquellas personas que se han instalado en él recientemente. A menudo, cuando se estudian los procesos de transformación social del territorio, y particularmente la gentrificación, se suelen presentar como grupos antagónicos. Las entrevistas realizadas en este estudio muestran las similitudes entre los dos grupos en la valoración que hacen de la evolución de la vivienda y del barrio. La sensación de inseguridad residencial es común en el conjunto de las personas entrevistadas, y esto se traslada a las dificultades a la hora de pensar y planificar proyectos de vida y familia a medio y largo plazo. El temor a quedarse sin hogar y tener que abandonar el barrio donde se ha vivido y se quiere seguir viviendo es fuente de malestar y de generación 
de situaciones de estrés y miedo (Novoa et al., 2014, Vásquez-Vera et al., 2016). Evidentemente, no queremos extender estas conclusiones al conjunto de la población que reside en el barrio desde hace poco o que lleva más tiempo viviendo en él. Las dificultades para acceder a determinados grupos con rentas y perfiles socioprofesionales altos han sido evidentes durante el trabajo de campo.

Y si los aspectos personales y la forma en la que impactan en términos de salud física y mental están siendo ampliamente recogidos por algunos estudios (Vásquez-Vera et al., 2017; 2019), en el caso que hemos analizado también quedan patentes los efectos negativos que dicho proceso está comportando sobre el propio tejido social y comunitario del barrio. Un elemento importante también para comprender el concepto de seguridad en su totalidad. A la inseguridad residencial se le añade la inseguridad comunitaria, en el sentido de la pérdida que experimenta la población residente y la que ya se marchó de sus lazos sociales. Las reformas urbanas y de servicios que se han puesto recientemente en marcha en el barrio mejoran las condiciones de vida de sus habitantes. El reto es poder seguir mejorando la calidad de vida sin el riesgo que comporta la expulsión de población a través de un mercado de la vivienda de compra y alquiler que se hace inasequible para una gran parte de la población del barrio que quiere seguir viviendo allí. No es fácil resolver el tema si no se encara desde una decidida política de control del precio de alquiler y de la dotación de un mayor parque de vivienda con algún tipo de protección social que permita regular los precios.

El trabajo de campo y el estudio pormenorizado que hemos desarrollado en Sant Antoni nos han permitido observar cómo se están viviendo las transformaciones sociales en el barrio, así como la expulsión de los grupos más vulnerables. Este tipo de proceso se podría hacer extensivo a otras zonas del municipio, ya que se ha constatado que estas dinámicas se han difundido a diversos barrios de la ciudad central, áreas que habían permanecido al margen de procesos previos de gentrificación y que han experimentado una fuerte revalorización en los últimos años (López-Gay et al., 2019). Un estudio de caso local que nos permite conocer procesos de escala global que impactan, como hemos podido constatar, en la vida cotidiana y en el bienestar de los residentes de nuestras ciudades.

\section{Agradecimientos}

Queremos agradecer a todos los vecinos y vecinas que han vivido o continúan viviendo en el barrio su buena predisposición a ayudarnos y atendernos durante el trabajo de campo. También mostramos nuestro agradecimiento a las organizaciones que trabajan en el barrio y en toda la ciudad de Barcelona por su colaboración prestada, en especial a la Associació de Veïns i Veïnes de Sant Antoni y al colectivo Fem Sant Antoni. Y por último, a los dos evaluadores/ as anónimos y los editores de la revista, que han leído el texto y han ayudado a mejorarlo. 


\section{Referencias bibliográficas}

Blanco-Romero, Asunción; Blázquez-Salom, Macià y Cànoves, Gemma (2018). "Barcelona, housing rent bubble in a tourist city. Social responses and local policies». Sustainability, 10 (6). <https://doi.org/10.3390/su10062043>

Bolívar Muñoz, Julia; Bernal Solano, Mariola; Mateo Rodríguez, Inmaculada; Daponte Codina, Antonio; Escudero Espinosa, Cecilia; Sánchez CantaleJo, Carmen et al. (2016). «The health of adults undergoing an eviction process». Gaceta Sanitaria, 30 (1), 4-10.

Bosch Meda, Jordi (2019). «La burbuja residencial en Europa (2000-2015). Dimensión y tipos». Documents d'Anàlisi Geogràfica, 65 (1), 11-41. <https://doi.org/10.5565/rev/dag.453>

Burgard, Sarah; Seefeldt, Kristin S. y Zelner, Sarah (2012). "Housing instability and health: findings from the Michigan Recession and Recovery Study». Social Science \& Medicine, 75 (12), 2.215-2.224. <https://doi.org/10.1016/j.socscimed.2012.08.020>

Cairney, John y Boyle, Michael (2004). «Home ownership, mortgages and psychological distress». Housing Studies, 19 (2), 161-174. <https://doi.org/10.1080/0267303032000168577>

Campbell, Iain; Parkinson, Sharon y Wood, Gavin (2013). «The housing security consequences of underemployment». AHURI Positioning Paper, 152, 1-72.

Chan, Sharni y Tweedie, Dale (2015). «Precarious Work and Reproductive Insecurity». Social Alternatives, 34 (4), 5-13.

Cócola Gant, Agustín (2016). «La producción de Barcelona como espacio de consumo. Gentrificación, turismo y lucha de clases». En: Grupo de Estudios Antropológicos La Corrala (coord.). Cartografía de la ciudad capitalista. Transformación urbana y conflicto social en el Estado español, 31-55. Madrid: Traficantes de Sueños.

Consejo Superior del Poder Judicial (CSPJ) (2013). "Aproximación a la conciliación de los datos sobre ejecuciones hipotecarias y desahucios». Boletín Información Estadistica, 35, 1-9.

DeIDdA, Manuela (2015). «Economic hardship, housing cost burden and tenure status: Evidence from EU-SILC». Journal of Family and Economic Issues, 36 (4), 531-556. <https://doi.org/10.1007/s10834-014-9431-2>

DownING, Janelle (2016). "The health effects of the foreclosure crisis and unaffordable housing: A systematic review and explanation of evidence». Social Science \& Medicine, 162, 88-96.

$<$ https://doi.org/10.1016/j.socscimed.2016.06.014>

Dupuis, Ann y Thorns, David C. (1998). «Home, home ownership and the search for ontological security». The Sociological Review, 46 (1), 24-47. <https://doi.org/10.1111/1467-954x.00088>

Esping Andersen, Gosta (1990). The three worlds of welfare capitalism. Princenton: Princenton University Press.

Eurofound (European Foundation for the Improvement of Living and Working Conditions) (2012). Quality of Life in Europe: Impacts of Crisis.

FIELDS, Desiree y UfFER, Sabina (2014). «The financialisation of rental housing: a comparative analysis of New York City and Berlin». Urban Studies, 53 (7), 1.486-1.502. <https://doi.org/10.1177/0042098014543704> 
Flaquer, Lluís (2004). "La articulación entre familia y el Estado de bienestar en los países de la Europa del sur». Papers. Revista de Sociologia, 73, 27-58. $<$ https://doi.org/10.5565/rev/papers/v73n0.1105>

Foessa (2012). Exclusión y desarrollo social. Madrid: Cáritas Española.

Garcia-López, Miquel-Àngel; Jofre-Monseny, Jordi; Martínez Mazza, Rodrigo y SEgú, Mariona (2019). "Do Short-Term Rental Platforms Affect Housing Markets? Evidence From Airbnb in Barcelona». IEB Working Paper, 2019/05. <https://doi.org/10.2139/ssrn.3428237>

Hernández Cordero, Adrián (2016). «De la botiga a la boutique. Gentrificación comercial en el Casc Antic de Barcelona». URBS: Revista de Estudios Urbanos y Ciencias Sociales, 6 (1), 79-99. <https://doi.org/10.31644/ED.6.2016.a05>

Hernández Cordero, Adrián; López Gay, Antonio; Ortiz y Guitart, Anna (2016). "Implicacions socials de tres intervencions urbanes al Casc Antic de Barcelona: el mercat de Santa Caterina, el Pou de la Figuera i el Born Centre Cultural». Treballs de la Societat Catalana de Geografia, 81, 111-136. <https://doi: 10.2436/20.3002.01.103>

Hiscock, Rosemary; Kearns, Ade; Macintyre, Sally y Ellaway, Anne (2001). "Ontological security and psycho-social benefits from the home: Qualitative evidence on issues of tenure». Housing, Theory and Society, 18 (10), 50-66. <https://doi.org/10.1080/140360901750424761>

Hulse, Kath y Haffner, Marietta (2014). «Security and Rental Housing: New Perspectives». Housing Studies, 29 (5), 573-578. <https://doi.org/10.1080/02673037.2014.921418>

Hulse, Kath y Saugeres, Lise (2008). «Housing insecurity and precarious living: An Australian exploration». AHURI Final Report, 124, 1-51.

KEMENY, Jim (1995). From public housing to the social market: rental policy strategy in comparative perspective. Londres: Routledge.

LoNG, Larry (1991). "Residential mobility differences among developed countries». International Regional Science Review, 14 (2), 133-147. <https://doi.org/10.1177/016001769101400202>

López Colás, Julián; Botelho de Azevedo, Alda y Módenes, Juan Antonio (2018). "Análisis de la diversidad de la inseguridad residencial». En: XVI Congreso de la Población Española. Alicante.

López-GaY, Antonio (2016). «Atracción de talento y polarización socioeconómica en Barcelona». Perspectives Demogràfiques. CED.

- (2017). «Hacia un patrón territorial complejo de la movilidad residencial. El caso de la Región Metropolitana de Barcelona». Papers. Revista de Sociologia, 102 (4), 793-823.

$<$ https://doi.org/10.5565/rev/papers.2420>

- (2018). "Cambio en la composición social y gentrificación en Barcelona: una mirada a través de los flujos migratorios y residenciales». Papers: Regió Metropolitana de Barcelona, 60, 80-93.

López-Gay, A.; Sales, J.; Solana, M.; Fernández, A.; Peralta, A. (2019). «Midiendo los procesos de gentrificación en Barcelona y Madrid: una propuesta metodológica». En: XIII CTV 2019 Proceedings: XIII International Conference on Virtual City and Territory: "Challenges and paradigms of the contemporary city". Barcelona: UPC.

$<$ https://doi.org/10.5821/ctv.8680> 
Lu, Max (1999). «Do people move when they say they will?: Inconsistencies in individual migration behavior». Population and Environment, 20 (5), 467-488. <https:// doi.org/10.1023/A:1023365119874>

Mansilla, José A. y Milano, Claudio (2019). «Becoming centre: tourism placemaking and space production in two neighbourhoods in Barcelona». Tourism Geographies. $<$ https://doi.org/10.1080/14616688.2019.1571097>

Módenes, Juan Antonio (1998). Flujos espaciales e itinerarios biográficos: La movilidad residencial en el área de Barcelona. Tesis doctoral. Universitat Autònoma de Barcelona.

- (2015). «Cambio demográfico, formación de hogares y sistema residencial». En: Torres Albero, C. et al. (eds.). Situación social España 2015, 127-138. Madrid: CIS.

- (2017). «La inseguridad residencial por problemas económicos en España comparada con el entorno europeo». Papers. Revista de Sociologia, 102 (4), 673-703. $<$ https://doi.org/10.5565/rev/papers.2416>

Módenes, Juan Antonio y Botelho-Azevedo, Alda (2017). «Más alquiler, ¿también más inseguridad residencial? Nuevas tendencias en los hogares jóvenes españoles tras la crisis». Revista de Estudios de Juventud, 116, 95-109.

Módenes, Juan Antonio y López-Colás, Julián (2014). «Movilidad residencial, trabajo y vivienda en Europa». Scripta Nova: Revista Electrónica de Geografía y Ciencias Sociales, 159.

Mora-Sanguinetti, Juan S. y Rubio, Margarita (2014). «Recent reforms in Spanish housing markets: An evaluation using a DSGE model». Economic Modelling, 44 (1), 42-49. <https://doi.org/10.1016/j.econmod.2014.04.028>

Moreno Mínguez, Almudena (2016). «Economic crisis and the new housing transitions of young people in Spain». International Journal of Housing Policy, 16 (2), 1-19. <https://doi.org/10.1080/14616718.2015.1130604>

Nettleton, Sarah y Burrows, Roger (1998). «Mortgage debt, insecure home ownership and health: An exploratory analysis». Sociology of Health and Illness, 20 (5), 731-753. <https://doi.org/10.1111/1467-9566.00127>

Novoa, Ana M.; Bosch, Jordi; Díaz, Fernando; Malmusi, Davide; Darnell, Mercè y Trilla, Carme (2014). «El impacto de la crisis en la relación entre vivienda y salud. Políticas de buenas prácticas para reducir las desigualdades en salud asociadas con las condiciones de vivienda». Gaceta Sanitaria, 28 (1), 44-50. <https://doi.org/10.1016/j.gaceta.2014.02.018>

Ortega, Eva; Rubio, Margarita y Thomas, Carlos (2011). «House purchase versus rental in Spain». Documentos de Trabajo. Banco de España, 1.108. <https://doi.org/10.2139/ssrn.1816329>

PAH (Plataforma de Afectados por la Hipoteca) y ASPB (Agència de Salut Pública de Barcelona) (2018). Voces y miradas. Inseguridad residencial y salud. Barcelona.

Poggio, Teresio (2008). "The intergenerational transmission of home ownership and the reproduction of the familialistic welfare regime». En: Saraceno, C. (ed.). Families, Ageing and Social Policy. Intergenerational Solidarity in European Welfare States, 59-87. <https://doi.org/10.4337/9781848445147.00009>

Sorando, Daniel y Leal, Jesús (2019). «Distantes y desiguales: el declive de la mezcla social en Barcelona y Madrid». Revista Española de Investigaciones Sociológicas, 167, 125-148. <https://doi.org/10.5477/cis/reis.167.125> 
TsAI, Alexander C. (2015). «Home foreclosure, health, and mental health: a systematic review of individual, aggregate, and contextual associations». PLoS One, 10 (4). <https://doi.org/10.1371/journal.pone.0123182>

VÁsqueZ-VERA, Hugo (2019). La inseguridad residencial por motivos económicos y su relación con la salud. Tesis doctoral. Universitat Pompeu Fabra.

Vásquez-Vera, Hugo; Fernández, Ana; Novoa, Ana M.; Delgado, Lucía; Barcala, Joaquín; Macías, Carlos y Borrell, Carme (2019). «Our lives in boxes: Perceived community mediators between housing insecurity and health using a Photovoice approach». International Journal for Equity in Health, 18 (1). <https://doi.org/10.1186/s12939-019-0943-0>

Vásquez-Vera, Hugo; Palència, Laia; Magna, Ingrid; Mena, Carlos; Neira, Jaime; Borrell, Carme (2017). "The threat of home eviction and its effects on health through the equity lens: A systematic review». Social Science and Medicine, 175, 199-208. <https://doi.org/10.1016/j.socscimed.2017.01.010>

VÁsquez-Vera, Hugo; Rodríguez-Sanz, Maica; Palència, Laia y Borrell, Carme (2016). «Foreclosure and Health in Southern Europe: Results from the Platform for People Affected by Mortgages». Journal of Urban Health, 93 (2), 312-330. <https://doi.org/10.1007/s11524-016-0030-4>

Wijburg, Gertjan; Aalbers, Manuel B.; Heeg, Susanne (2018). «The financialisation of rental housing 2.0: releasing housing into the privatised mainstream of capital accumulation». Antipode. A Radical Journal of Geography, 50 (1), 1.098-1.119. <https://doi.org/10.1111/anti.12382> 